Proc. Indian Acad. Sci. (Chem. Sci.), Vol. 90, Number 1, January 1981, pp. 19-26.

C) Printed in India.

\title{
Nature of dependence of spin-orbit splittings on atomic number*
}

\section{D SARMA}

Solid State and Structural Chemistry Unit, Indian Institute of Science, Bangalore 560012 , India

MS received 28 August 1980 ; revised 7 October 1980

\begin{abstract}
It has been shown that Dirac equation employing a constant value of the screening constant $Z_{0}$ does not explain the variation of spin-orbit splittings of $2 p$ and $3 p$ levels with atomic number $Z$. A modef which takes into account the variation of $Z_{0}$ with $Z$ is shown to satisfactorily predict the dependence of spinorbit splittings on $Z$.
\end{abstract}

Keywords. Spin-orbit splittings; Dirac equation; screening constant.

\section{Introduction}

Spin-orbit coupling and the consequent lifting of the degeneracy of the two $J$-multiplets of a singly occupied atomic orbital are described by solutions of Dirac's relativistic wave equation (Dirac 1958). The resulting spin-orbit splitting, $\triangle E_{n l}\left(=E_{n l_{1}}-E_{n l j_{2}}\right)$, for the hydrogenic case is given by

$$
\begin{aligned}
\Delta E_{n l}= & m c^{2}\left[\frac{\left(k_{1}^{2}-\gamma^{2}\right)^{1 / 2}+n_{1}}{\left\{k_{1}^{2}+n_{1}^{2}+2 n_{1}\left(k_{1}^{2}-\gamma^{2}\right)^{1 / 2}\right\}^{1 / 2}}\right. \\
& \left.-\frac{\left(k_{2}^{2}-\gamma^{2}\right)^{1 / 2}+n_{2}}{\left\{k_{2}^{2}+n_{2}^{2}+2 n_{2}\left(k_{2}^{2}-\gamma^{2}\right)^{1 / 2}\right\}^{1 / 2}}\right] .
\end{aligned}
$$

Here, $y=a Z, a$ being the fine structure constant, $Z$ the atomic number; $k$ and $n$ are quantum numbers, the subscripts 1 and 2 denoting the two different $J$-valued spin-orbit levels. For systems containing more than one electron (non-hydrogenic case), the effect of other electrons can be accounted for by replacing the nuclear charge $Z$ by an effective charge $\left(Z-Z_{0}\right)$, where $Z_{0}$ represents the screening due to other electrons. In the non-relativistic limit, the above equation can be approximated as,

$$
\Delta E_{n l}=\frac{m c^{2}}{2 n^{3}}\left\{\frac{1}{\left|k_{1}\right|}-\frac{1}{\left|k_{2}\right|}\right\} a^{4}\left(Z-Z_{0}\right)^{4}
$$

\footnotetext{
* Communication No. 98 from the Solid State and Structural Chemistry Unit.
} 
This equation still uses the spherical coulombic potential with an effective $\left(Z-Z_{0}\right) e$ charge at the nucleus. Small deviations from the spherical nature of the potential can be accommodated by writing equation (2) as,

$$
\triangle E_{n l}=\mathbf{a}\left(Z-Z_{0}\right)^{4}
$$

where $\mathbf{a}$ is the parameter accounting for the non-sphericity of the potential. Thus (1) and (3) have been employed to understand the dependence of experimental $\triangle E_{n l}$ values (from $x$-ray photoelectron spectroscopy) on $Z$ in related series of metal oxides (Sarma and Rao 1980). These equations (with a constant $Z_{0}$ value) could not, however, explain why $\triangle E_{2 p}$ in transition metal oxides $(Z=22-29)$ vary linearly with the atomic number (Rao et al 1979), the difference between the experimental and the calculated values of $\Delta E_{2}$, in these systems being much beyond the experimental error. Large deviations of experimental $\triangle E_{2 p}$ from values calculated from (1) have also been found in transition metals (Barrie et al 1974). Such deviations are noticed (Sarma and Rao 1980) for other levels (e.g., $3 d$ and $4 d$ ) in the case of metal oxides over narrow ranges of $Z$. These deviations cannot be explained on the basis of (1) and (2) which employ a constant $Z_{0}$ value for all elements for a given level $(n l)$. It, therefore, becomes necessary to find a functional relation between $Z_{0}$ and $Z$ which leads to satisfactory results for $\triangle E_{n l}$ over the entire range of $Z$ values. In this paper, we have shown how the screening constant $Z_{0}$ varies with $Z$ due to various electron-electron interactions and then, compared the observed $\triangle E_{n l}$ values with those predicted by equation (1) incorporating a $Z_{0}(Z)$ term based on our model. The results are indeed most encouraging and provide an insight into the nature of screening.

\section{Methodology}

Using the experimental values of $\triangle E_{2 p}$ and $\triangle E_{3 p}$ from the literature (Bearden and Burr 1967), $Z_{0}$ values for the elements were calculated from equation (1), the error in $Z_{0}$ being estimated from the errors in the values of the spin-orbit splittings. The uncertainties in $Z_{0}$ values were rather large in the case of $2 p$ levels for elements with $Z<20$ and $Z>75$; for the $3 p$ level, it was large for elements with $Z<38$ and $Z>83$ and also for $Z=57-70$. We, however, retained $\triangle E_{3 p}$ of $\operatorname{Ar}(Z=18)$ as this value is known with great accuracy from UV photaelectron spectroscopy. In the region of $Z=11-19$, the $Z_{0}$ value is extremely sensitive to small changes in the $\triangle E_{2 p}$ values; although the absolute error of $\triangle E_{2,}$ over this region is not large, it leads to large errors in $Z_{0}$ values and thus, this region was not included in the data set for the $2 p$ level. The $Z_{0}$ values thus obtained are plotted against $Z$ in figures 1 and 2 for the $2 p$ and $3 p$ levels respectively. The figures clearly illustrate how $Z_{0}$ varies markedly when we consider a wide range of elements; furthermore, the nature of variation of $Z_{0}$ varies from one level to another.

In order to provide a simple heuristic model, we propose that there are three types of interactions that should be taken into account to explain the variation of screening constant with $Z$. These contributions are: (i) screening due to electrons closer to the nucleus than the level in question, (ii) coulombic contribution of outer electrons to screening and (iii) spin-spin interaction of the core 


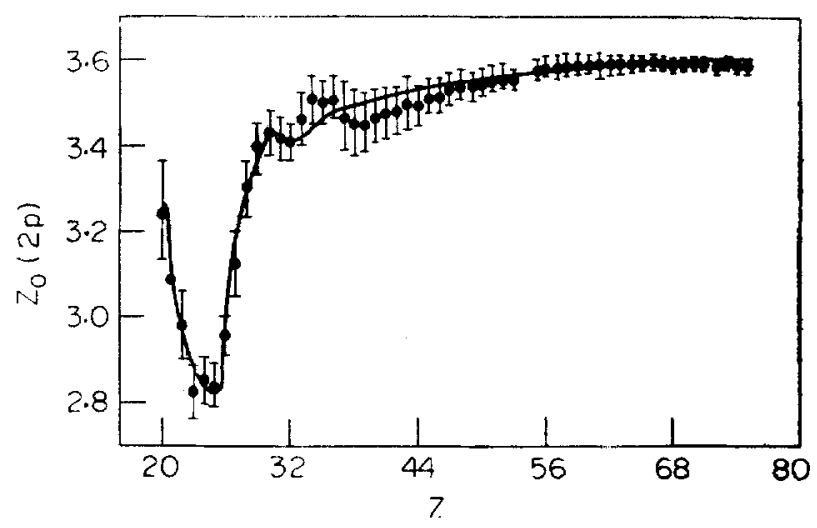

Figure 1. Plot of $Z_{0}(2 p)$ against $Z$. The solid line is the least-square fit to the data points according to equation (4).

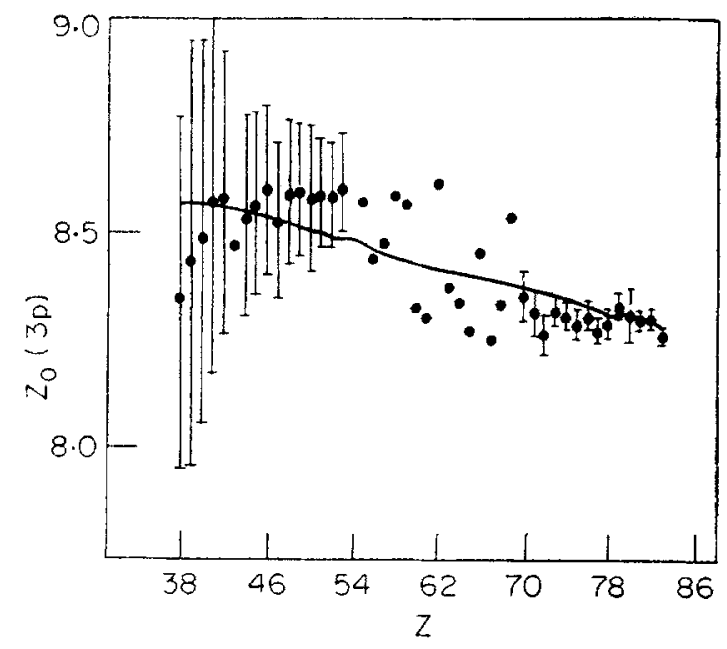

Figure 2. Plot of $Z_{0}(3 p)$ against $Z$. The errors in $Z_{0}$ values are shown for the points which have been used for the least-square fit procedure; for other $Z_{0}$ values, the errors are very large. The solid line is the least-square fit according to equation (4) with only the first two terms.

hole with the outermost open shell. The largest contribution would be duc to (i) and would vary from one level to another; we shall denote this by a constant $Z_{0}^{s}$, since this contribution essentially represents screening as is usually understood. Contribution from (ii) would involve electron-electron interaction including direct and exchange terms and would vary with the number of outer clectrons. Since it is impossible to depict the functional relationship of these interactions on the outer electrons in a closed form, we have arbitrarily chosen

$$
\sum_{i} n_{j}^{i}\left(E_{n l}^{i}{ }^{3}-E_{j}^{i}\right)^{j}
$$


to represent the effect of the interactions, where $j$ denotes an outer level, $n_{j}^{i}$ the occupancy of the level $j$ in the $i$-th element, $E_{n i}^{i}$ and $E_{j}^{i}$ are the energies of the $(n l)$ and $j$ levels and $X$ is a negative number. Similarly, we have chosen to represent the spin-spin interaction by

$$
S_{\mathrm{v}}^{i} \exp \left[\left(E_{n i}^{i}-E_{v}^{i}\right) / y\right]
$$

where $S_{v}^{i}$ is the spin due to unpaired electrons in the outermost valence level, $v$, of the $i$-th element, $E$ s are the energies and $y$ is a negative number. The weighting factors $\left(E_{n l}^{i}-E_{y}^{i}\right)^{x}$ and $\exp \left[\left(E_{n b}^{i}-E_{v}^{i}\right) / y\right]$ in these two interactions are chosen to be different because of the different spatial dependence of these two interactions. Both these factors approach a value of zero as the energy separation [between the levels $(n l)$ and $j$ or $v$ ] becomes larger representing the limiting behaviour of these interactions correctly. The factor $\left(E_{n i}^{i}-E_{j}^{i}\right)^{x}$ diverges as $E_{j}^{i}$ approaches the value of $E_{n l}^{i}$ in order to simulate the decreasing separation between the electrons from two levels; this represents the divergence of coulombic interaction with decreasing separation. The exponential factor, exp $\left[\left(E_{n l}^{i}-E_{v}^{i}\right) / y\right]$, in the spin-spin interaction term has a limiting value of unity as $E_{v}^{i}$ approaches $E_{n l}^{i}$ and thus, simulates this interaction as the electrons with the same spin are forbidden to approach each other very closely due to Pauli's exclusion principle. Taking all these three terms together, the total screening constant $Z_{0}$ can be written as,

$$
Z_{0}=Z_{0}^{s}+A \sum_{j} n_{j}^{i}\left(E_{n t}^{i}-E_{j}^{i}\right)^{x}+B S_{\mathrm{r}}^{i} \exp \left[\left(E_{n t}^{i}-E_{\mathfrak{v}}^{i}\right) / y\right]
$$

In (4) we would expect $A$ to be positive and $B$ to be negative as the electronelectron coulombic interaction is repulsive whereas the spin-spin interaction stabilises the electronic state.

The various parameters in (4) were calculated for the $2 p$ level by a least-square fit (root mean square deviation $=0.022$, correlation coefficient $=0.994$ ) of the $Z_{0}$ values obtained from experimental $\triangle E_{2}$ data (employing equation 1). The parameters so obtained are as follows: $Z_{i}^{s}=2.6529, \quad A=0.6685, \quad B=$ $-1 \cdot 15946, x=-0.4122$ and $y=-384 \cdot 5$. It is interesting to note that the signs of $A$ and $B$ come out as expected, the latter showing a negative sign. These parameters were then used to calculate the $Z_{0}$ versus $Z$ curve shown in figure 1 . The marked decreasz in $Z_{0}$ in the region of $Z=25$ is due to the spin-spin interaction term; the electron-electron repulsion term would only give a smaoth increase from $Z_{0}=3.25$ to $Z_{0}=3.5$ in the region $Z=20-40$. The spin-spin interaction term becomes dominant in the region of first-row transition metals while it is negligible for $Z>40$ due to negligible overlap between the core hole and the valence level in this region.

The $Z_{0}$ values of $3 p$ level obtained from experimental $\triangle E_{3 n}$ values (figure 2) exhibits a behaviour entirely different from $Z_{0}(2 p)$ values. In view of the large uncertainties in these $Z_{0}(3 p)$ data, we considered it appropriate to omit the spinspin interaction term in (4), since it would only account for small variations. The parameters obtained by the least-square fit of (4) with the first two terms 
alone (root mean square deviation $=0.072$, correlation coefficient $=0.956$ ) are as follows:

$$
Z_{0}^{s}=7 \cdot 26072, A=1 \cdot 22719 \text { and } x=-0.5615 \text {. }
$$

We see that $Z_{0}^{:}$value of $3 p$ is larger than that for $2 p$ as expected. The value of $Z_{0}$ for $\operatorname{Ar}(7 \cdot 26)$ calculated from these parameters is indeed very close to the value of $Z_{0}$ obtained from experimental $\triangle E_{3 p}$ value $(7 \cdot 28)$. The calculated value of $\triangle E_{3 \mathrm{p}}$ in the case of $\mathrm{Cl}^{-}$is also close to the observed value $(\sim 0 \cdot 1 \mathrm{eV})$. We should, however, note that spin-spin interaction may, indeed, become very important in the region of $Z=19-37$ giving rise to a significant decrease in $Z_{0}$ values for these elements. If good data for $\triangle E_{3 y}$ were available, we feel certain that equation (4) with spin-spin interaction term would predict the exact variation of experimental $Z_{0}$ values.

\section{Results and discussion}

In figures 3 and 4 we have shown how the values of spin-orbit splittings for $2 p$ and $3 p$ levels calculated from the Dirac equation [equation (1)] employing $Z_{0}$

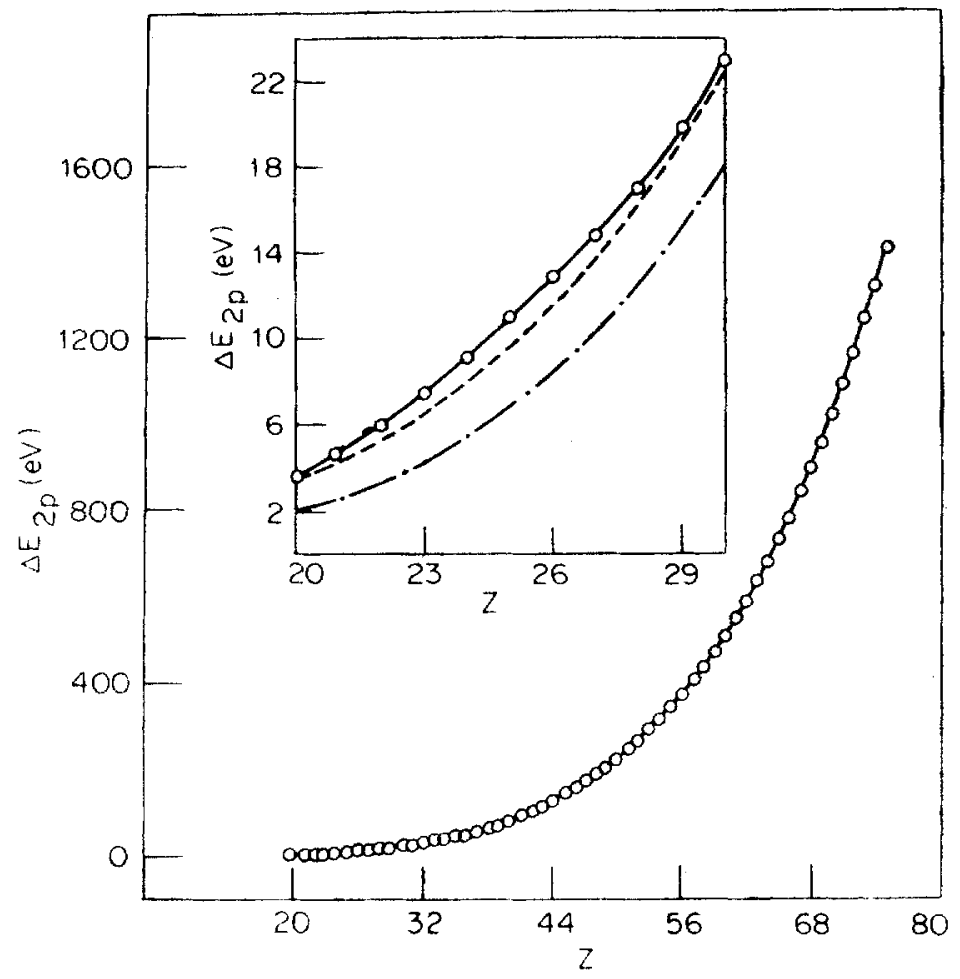

Figure 3. Plot of $\triangle E_{2 p}$ values against atomic number $Z$. The solid line is the calculated plot of $\triangle E_{2 p}$ using Dirac equation incorporating $Z_{0}(Z)$ according to equation (4). The inset shows the comparison between the values of $\triangle E_{2, j}$ predicted according to equation (1) (---), equation (3) (-., - ) with constant $Z_{0}$ and the present model (the solid line); the experimental values are shown as solid circles. 


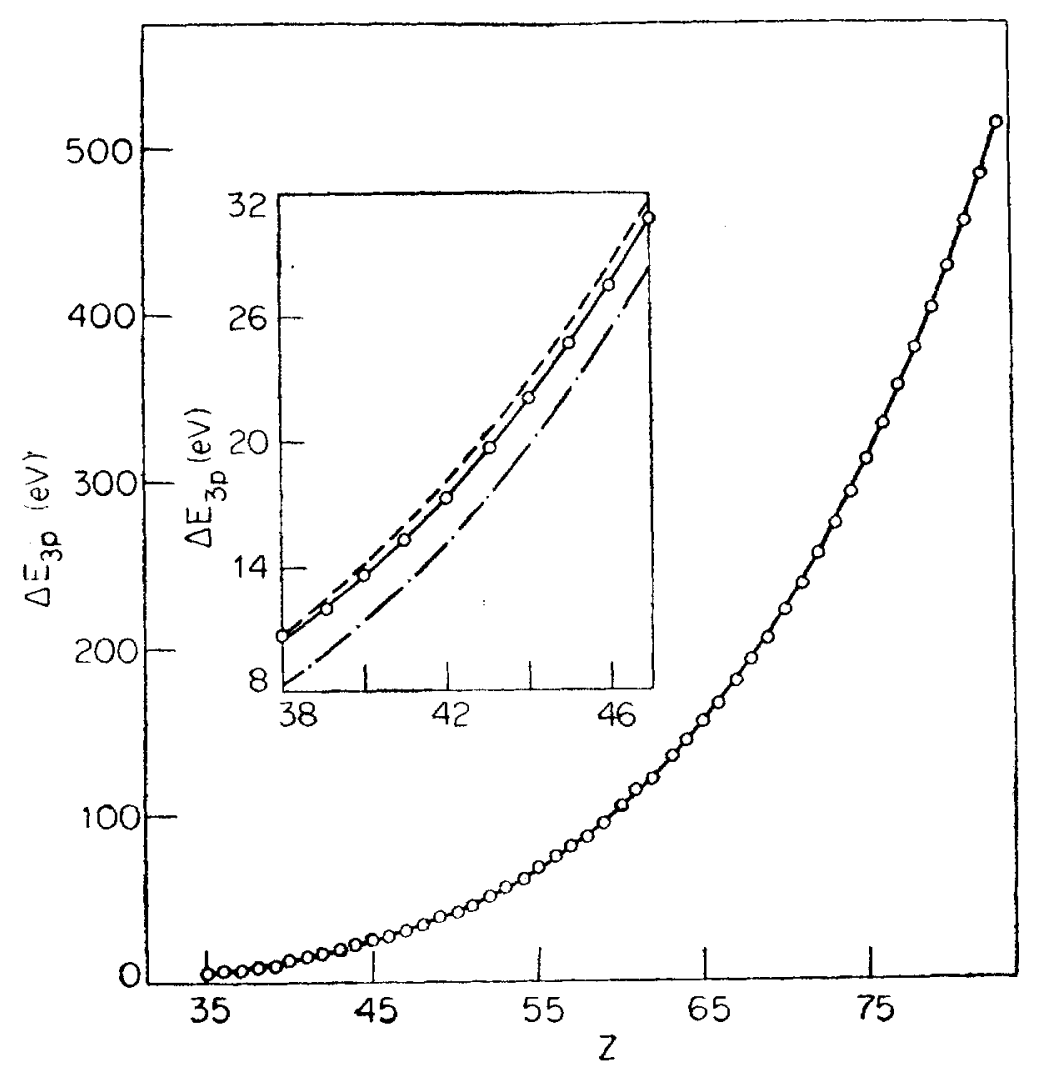

Figure 4. Plot of $\triangle E_{3 p}$ values against $Z$. The solid line is the calculated values of $\triangle E_{3 p}$ according to the present model. The inset shows the comparison between the values of $\triangle E_{3 p}$ predicted according to equation (1) (-- - ), equation (3) (-.- - with constant $Z_{0}$ and the present model (the solid line) over a narrow range of $Z$; the experimental values of $\triangle E_{3_{p}}$ are shown as solid circles.

values given by (4) show excellent agreement with experimental data. In these figures, we have also shown how the description of $Z_{0}$ given in terms of (4) gives more reliable predictions of $\triangle E_{2 p}$ and $\triangle E_{3 p}$ than when we use (1) or (3) with constant $Z_{0}$ values. With constant $Z_{0}$ values (1) and (3) give much poorer fits for both $\triangle E_{2}$ ( $Z$ range, 20-75) and $\triangle E_{3}$ ( $Z$ range, 35-83) as can be seen from the root mean square deviations given in table 1.

The parameters of (4) for the $2 p$ level mentioned earlier were employed to calculate the spin-orbit splittings $\left(\triangle E_{2 p}\right)$ in some of the lighter elements $(Z=11-19)$. The calculated and experimental values are shown in table 2 . We clearly see the success of this model describing the total screening constant from this table.

The description of $Z_{0}$ given in (4) appears to be able to explain certain specific variations of spin-orbit splittings found in transition metal systems. Thus, (4) predicts that transition metal ions like $\mathrm{Fe}^{2+}, \mathrm{Co}^{2+}$ and $\mathrm{Co}^{3+}$ should have a higher $\triangle E_{2,}$ when they are in the high-spin state than in the low-spin state. The predicted difference varies between 0.4 and $0.8 \mathrm{eV}$ compared to the experimental values which show differences of $0.4-0.6 \mathrm{eV}, \triangle E_{2 \beta}$ values calculated for $\mathrm{Cr}_{2} \mathrm{O}_{8}$ and 
Table 1. Root mean square deviations in least-square fits of $\triangle E_{2 p}$ and $\triangle E_{3 p}$ values employing different equations.

\begin{tabular}{ccccc}
\hline Level & Range of $\boldsymbol{Z}$ & Equation (1)(a) & Equation (3)(a) & Present model \\
\hline $2 p$ & $20-75$ & 0.771 & 5.400 & 0.432 \\
$3 p$ & $35-83$ & 1.094 & 2.133 & 0.803 \\
\hline
\end{tabular}

(a) The value of constant $Z_{0}$ which gave minimum deviation is used in the least-square fit.

Table 2. Calculated and experimental spin-orbit splitting values (in $\mathrm{eV}$ ) for some elements.

\begin{tabular}{|c|c|c|c|}
\hline$Z$ & Element & $\begin{array}{c}\text { Calculated } \\
\qquad \triangle E_{2 p}\end{array}$ & $\begin{array}{l}\text { Experimental } \\
\qquad \triangle E_{2,}{ }^{(a)}\end{array}$ \\
\hline 11 & $\mathrm{Na}$ & $0 \cdot 3$ & 0.3 \\
\hline 12 & $\mathrm{Mg}$ & $0 \cdot 3$ & $0 \cdot 3$ \\
\hline 13 & $\mathrm{Al}$ & 0.5 & 0.4 \\
\hline 14 & $\mathbf{S i}$ & 0.9 & $1 \cdot 0$ \\
\hline 15 & $\mathbf{P}$ & $1 \cdot 4$ & $1 \cdot 0$ \\
\hline 16 & $\mathbf{S}$ & 1.6 & $1 \cdot 0$ \\
\hline 17 & $\mathrm{Cl}$ & $1 \cdot 8$ & $2 \cdot 0$ \\
\hline 18 & $\mathrm{Ar}$ & $2 \cdot 2$ & $2 \cdot 1$ \\
\hline 19 & $\mathrm{~K}$ & $3 \cdot 0$ & $2 \cdot 7$ \\
\hline 19 & $\mathrm{~K}^{+}$ & $2 \cdot 8$ & $2 \cdot 7$ \\
\hline
\end{tabular}

(a) The experimental values of $\triangle E_{2 p}$ are taken from Shirley et al (1977).

$\mathrm{CrO}_{3}$ show a differences of about $0.4 \mathrm{eV}$ between the two compared to an observed difference of $0.8 \mathrm{eV}$. A lowering of $\triangle E_{2}$, by $0.7 \mathrm{eV}$ is predicted for the $d^{0}$ state of $\mathrm{Mn}\left(\mathrm{Mn}^{7+}\right)$ compared to that in $\mathrm{Mn}^{2+}$. An increase of $\triangle E_{2 p}$ by $0.2 \mathrm{eV}$ is predicted in going from $\mathrm{Mn}$ to $\mathrm{MnO}$, while the observed change is $0.4 \mathrm{eV}$. Equation (4) also predicts differences between spin-orbit splittings of elements and their compounds, but we would not expect a quantitative agreement in view of the differences in bonding, crystal field effects and so on.

\section{Acknowledgement}

The author is thankful to Professor C N R Rao for suggesting the problem and also for his kind help throughout the course of this work, 


\section{References}

Barrie A, Drummond I W and Herd Q C 1974 J. Electron. Spectrosc. Rel. Phenom. 5217 Bearden J A and Burr A F 1967 Rev. Mod. Phys. 39125

Dirac P A M 1958 Principles of quantum mechanics Ch. XI (Oxford University Press)

Rao C N R, Sarma D D, Vasudevan S and Hegde M S 1979 Proc. R. Soc. London A367 239

Sarma D D and Rao C N R 1980 J. Electron Spectrose. Rel. Phenom. 2025

Shirley D A, Martin R L, Kowalczyk S P, McFeely F R and Ley L 1977 Phys. Rev. B15 54 